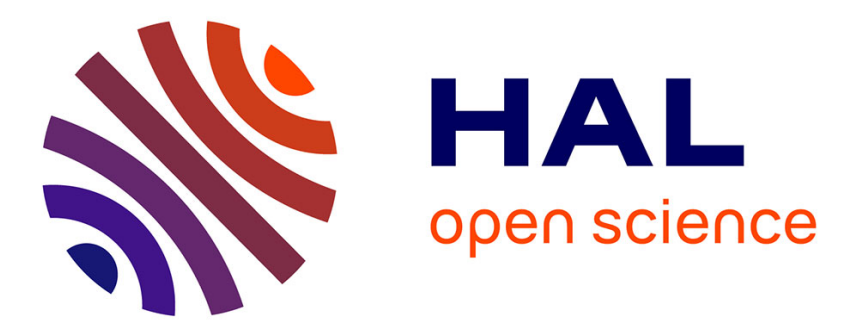

\title{
Access Selection and Joint Pricing in Multi-Operator Wireless Networks: A Stackelberg Game
}

\author{
Soha Farhat, Samhat Abed Ellatif, Samer Lahoud, Bernard Cousin
}

\section{To cite this version:}

Soha Farhat, Samhat Abed Ellatif, Samer Lahoud, Bernard Cousin. Access Selection and Joint Pricing in Multi-Operator Wireless Networks: A Stackelberg Game. The Fifth International Conference on Digital Information and Communication Technology and its Applications (DICTAP2015), Apr 2015, Beyrouth, Lebanon. pp.38 - 43, 10.1109/DICTAP.2015.7113167 . hal-01145999

\author{
HAL Id: hal-01145999 \\ https://hal.science/hal-01145999
}

Submitted on 27 Apr 2015

HAL is a multi-disciplinary open access archive for the deposit and dissemination of scientific research documents, whether they are published or not. The documents may come from teaching and research institutions in France or abroad, or from public or private research centers.
L'archive ouverte pluridisciplinaire HAL, est destinée au dépôt et à la diffusion de documents scientifiques de niveau recherche, publiés ou non, émanant des établissements d'enseignement et de recherche français ou étrangers, des laboratoires publics ou privés. 


\title{
Access Selection and Joint Pricing in Multi-Operator Wireless Networks: A Stackelberg Game
}

\author{
Soha Farhat ${ }^{1,2}$, Abed Ellatif Samhat ${ }^{1}$, Samer Lahoud ${ }^{2}$, Bernard Cousin ${ }^{2}$ \\ soha.farhat@ul.edu.lb, samhat@ul.edu.lb, samer.lahoud@irisa.fr, Bernard.Cousin@irisa.fr \\ ${ }^{1}$ Lebanese University, Ecole Doctorale des Sciences et Technologies, Hadath, Lebanon \\ ${ }^{2}$ University of Rennes I - IRISA, France
}

\begin{abstract}
We investigate in this paper the access selection and joint pricing problem in multi-operator wireless networks. The problem is formulated as a Stackelberg game, where cooperating service operators first set the service price to maximize their revenue. Then, the home operator of the mobile user performs the access selection process among the service operators, in order to maximize its own profits and its client satisfaction, in terms of perceived QoS. Competing operators decide the service price following a number of defined pricing schemes, and the home operator uses a hybrid utility function for the selection decision. We consider the Nash equilibrium as a solution of the service price setting game, where the best response is presented in function of the adopted pricing scheme. Simulation results show the efficiency of this multi-leader follower game for the access selection in a multi-operator environment, and illustrate how operators' cooperation enhances network performance and improves operators' revenue.
\end{abstract}

Keywords - Multi-operator sharing networks; cooperation, multi-leader follower game; access selection; service pricing; Nash equilibrium.

\section{INTRODUCTION}

The mobile traffic broadband is growing in a fast manner, facing network operators with the challenge of expanding capacity and enhancing the Quality of Service (QoS) of their communication networks. Multi-operators cooperation in the form of open access has been proposed in a number of large research projects like ANWIRE, SPICE, Ambient Networks, etc. [1]. The cooperation of wireless network operators in a sharing environment is also introduced in [2] as a cost effective network solution to expand capacity and improve operators' profitability. In fact, network sharing is widely used in WLAN systems where local access providers offer wireless access to service providers. A similar structure exists in cellular networks where Mobile Virtual Network Operator (MVNO) provides mobile services without having a mobile network of their own. Multi-operator solutions are introduced for shared small cells as for macro cellular networks, within different business models including inter-connection provider or a third party local operator [3] [4].

Indeed, current $3^{\text {rd }}$ Generation Partnership Project (3GPP) standards fully support network sharing between operators under different sharing schemes. With the latest advancements introduced in LTE-Advanced systems [5], spectrum sharing is feasible efficiently. Furthermore, new mobile architecture arises in order to help operator upgrading their networks, and enable network sharing in a Cloud Radio Access Network (CRAN). In C-RAN, baseband processing is centralized and shared among sites in a virtualized Baseband Units (BBU) Pool. The latter can be shared by different network operators, allowing them to rent Radio Access Network (RAN) as a cloud service [6].

In our work, we consider a cooperative environment where wireless network operators share their radio resources, to upgrade their networks' capacity and improve performance in terms of clients' rejection and QoS degradation. In this multioperator environment, when an operator is unable to satisfy his client, he tries to give him access to the service through another network operator, thus avoiding his rejection. In addition, in order to preserve the transparency of the transaction, the mobile user payment goes to his home operator, and the latter must pay a service price (transaction cost) to the new access operator. This cooperation model was introduced first in [7] and [8].

In this paper, we model the interaction between the wireless operators as a multi-leader-follower (Stackelberg) game. Available service operators announce their transaction cost in the first stage and the home operator of the transferred user performs the selection decision in the second stage. The decision of the home operator is based on the service operators' transaction cost (service price) and the delivered QoS parameters. Service operators select the service price that maximizes their revenue, keeping in mind the impact of their prices on the home operator profit gain. For a service operator the best response is the price scheme maximizing his global profits.

The rest of the paper is organized as follows: Section II presents some existing work related to access selection and joint pricing using game theory. Section III presents our multileader-follower game and the adopted pricing schemes are described in section IV. Section V shows simulation results for the efficiency of the selection decision using our Stackelberg game formulation, and elaborates the optimal pricing scheme for the transaction cost decision. Finally, we conclude in section VI.

\section{BACKGROUNG AND RELATED WORKS}

In a multi-operator sharing environment, two important issues are to be studied: Access selection and joint service pricing. For the access selection, a hybrid decision must achieve the highest revenue for the network operator while 
guaranteeing the maximum satisfaction of the mobile users from the service usage. Accordingly, various mathematical tools can be used as for the selection decision in single operator networks [9-12], such as utility and cost function theory [78][13], multiple attribute decision making (MADM) [7], fuzzy logic [14-15] and game theory[13][17-20]. Moreover, operators compete with each other to offer wireless access services through price adjustment. In general, competitive pricing is formulated as a non-cooperative game, and the most popular solution is the Nash equilibrium concept [17-20].

Besides, most of the existing works in multi-operator network environment use game theory for the access selection and the joint service pricing. In [17], authors applied a noncooperative game using Leader-follower model (Stackelberg game) in order to study the competition between two ISPs. With a simple QoS model a Nash equilibrium point was found from which the two ISPs would not move without cooperation. Leader-follower game model is used again in [18] for competitive pricing in heterogeneous wireless access networks, between the WiMAX and WiFi service providers. Authors showed that when competing service providers make their decision in a simultaneous-play the best response can be obtained as in the Bertrand game model with the Nash equilibrium. But, when a service provider is defined as a leader and it makes the price decision before the other service providers, the solution moves to the Stackelberg equilibrium which is always the best response of the follower service providers. In cognitive radio networks [19], Nash equilibrium concept is used to find the optimal price in a Stackelberg game between primary and secondary operators and Wardrop equilibrium is determined for the network selection game. Authors reveal the advantage for primary operator to play before the secondary operator, particularly in a high-traffic regime. Furthermore, a two-stage multi-leader-follower game is used to model the interaction of a number of wireless providers and a group of atomic users in [20]. Authors showed that the provider competition leads to a unique socially optimal resource allocation for a broad class of utility functions and a generic channel model. A new "flex service" paradigm is introduced in [13], it allows a mobile user to dynamically access base stations BSs of different providers based on various criteria, such as profile, network conditions and offered prices. "Flex users" can select the appropriate provider and BS on a per-session basis. Authors presented two modeling framework for the access markets at both microscopic and macroscopic levels. At macroscopic level, users are considered as a homogeneous population with respect to preferences and decision-making mechanism. In such model, the behavior of users is described by a population game in order to determine how the entire user population reacts to the decision of providers.

All works listed above for joint pricing and access selection consider the price that service provider or network operator offer for the mobile users. In this paper, we are studying the service price that operators set for the user transaction from one operator to another. This price is transparent for the users and it is charged to the home operator of a transferred user. The envisaged mobile market is more open than "Flex service" market for mobile users, since it does not need any previous subscription as a "Flex user". Besides, it is controlled by the home operator decision; the user is not free to switch between operators. Other works [14] [15] based on fuzzy theory for access selection, proposed different business models for price sharing.

In this paper we model the interaction between network operators as a Stackelberg game in order to find the optimal pricing scheme to adopt for the service price setting of interoperator users' transaction. The available service operators for the selection are considered as leaders and the home operator of the transferred user is the follower.

\section{STACKELBERG GAME FORMULATION}

We consider a simultaneous-play and a two stages leaderfollower game where $\boldsymbol{N}$ network operators are the players. In the first stage of the game, the strategy of a player is the service price per unit of bandwidth for the transaction cost (denoted by $C s_{i}$ ). The payoff for each player is its profit denoted by $\pi_{i}$. This profit is calculated based on the revenue obtained from own clients, the transaction cost and the revenue obtained from the guest users. A simulation framework similar to the microscopic level framework used in [12] and described in [4] is adopted to obtain the values of achieved payoff for each player. In the second stage of the game where prices $\mathbf{C s}=\left[\mathrm{Cs}_{1} \mathrm{Cs}_{2} \ldots \mathrm{Cs}_{\mathrm{N}-1}\right]$ are known, the home operator choose a service operator that maximizes its new payoff, which is the utility of the transferred user added to the transaction profit. This payoff is given by:

$$
U_{h}\left(C s_{i}\right)_{=} W_{u} * U_{u+} W_{o p} *\left(p-C s_{i}\right)
$$

Where, $U_{u}$ is the transferred user utility function. This utility function is a combination of different QoS parameters offered by an access network [16]. We use Simple Additive Weighting Method (SAW) to obtain it for non-real time service users as follow [16]:

$$
U_{u}=W_{J} * J_{M+} W_{D} * D_{M}+W_{B W} * B W_{R+} W_{B} * B E R_{M}
$$

And we use Multiplicative Exponential Weighting Method (MEW) for real time service users as follow [16]:

$$
U_{u}=J_{M}{ }^{W J} * D_{M}{ }^{W D} * B W_{R}{ }^{W B W} * B E R_{M}{ }^{W B}
$$

Given $J_{M}$ is the mean of the jitter, $D_{M}$ is the mean of the endto-end delay, $\quad B W_{R}$ is the remaining bandwidth and $B R_{M}$ is the mean of the loss rate measured on the service operator network. These parameters are normalized and associated to their corresponding preference weights $\mathrm{W}_{\mathrm{J}}, \mathrm{W}_{\mathrm{D}}, \mathrm{W}_{\mathrm{BW}}$ and $\mathrm{W}_{\mathrm{B}}$ respectively, associated to each service class. In addition, $p$ is the price that the transferred user pays for the home operator such that $(p-C s)$ is the home operator profit from the transaction. And, the weights $W u$ and Wop are the degree of important for the user satisfaction in terms of QoS and the operator satisfaction in terms of profits, respectively. 


\section{Equilibrium strategies}

Consider that Home operator faces Cs in stage II. This operator makes the decision for access selection in order to maximize $U_{h}(C s)$. The best response of this stage is to select the service operator that maximizes both the user and the home operator satisfaction in terms of QoS and profits respectively.

In stage I, the optimal choice of prices for the transaction cost depends on the home operator reaction and its selection decision. The best response of a service operator $i$ is the optimal price $C s_{i}{ }^{*}$ for which profit $\pi_{i}\left(C s_{i}{ }^{*}, C s j\right)$ is maximized given the price $C s_{j}$ offered by the other serving operator. When available service operators offer their prices simultaneously, Nash equilibrium gives the set of prices such that none of the operators can increase the profit by choosing a different price, given the price offered by the other service operator. For the set of price strategies, we consider six different pricing schemes described in the following section, the output of the game for each strategy is $\mathbf{C s}=\left[\mathrm{Cs}_{1} \mathrm{Cs}_{2} \ldots \mathrm{Cs}_{\mathrm{N}-1}\right.$ ]. A utility space $\left(\pi_{\mathrm{i}}, \pi_{\mathrm{j}}\right)$ is used to plot different payoff curves for each price strategy [18], in order to deduce the outcomes of the pricing game between each pair of operators.

\section{PRICING SCHEMES}

\section{A. Scheme S1}

To prevent any loss of investment, a guest user must generate the same revenue from a client. Thus, the transaction cost is set equal to the user price $C s_{i}^{l}=p_{i}$.

\section{B. Scheme S2:}

We may notice, in $S 1$, that an operator having the cheapest service price will pay a high price for its client transfer and gain less from guest users. It may cause losses in sporadic cases where this transfer is frequent. Thus, in this scheme, we propose that $C s^{2}=\max _{i}\left(p_{i}\right) i=1,2,3 \ldots$

\section{Scheme S3:}

In scheme S2 the price agreed for Cs guarantee more revenues from guest users but it causes losses for client transaction. To improve users' acceptance, an operator may perform a high rate of user's transaction, which causes a lot of charges in S2. Operators may find better to pay less and get less than pay more. Thus, S3 proposes a price $C s^{3}=\min _{i}\left(p_{i}\right)$ $i=1,2,3 \ldots$

\section{Price Sharing scheme ( pShare):}

With price sharing S-op takes a share from the payment of the transferred user. Thus, H-op will keep $(1-\alpha) p$, and S-op will get $\alpha p$, where $\alpha \geq 0$. Depending on the value of $\alpha$, different sub-schemes can be envisaged:

1) Scheme pShare1: In this scheme, $\alpha=1$, i.e, S-op gets all the revenue from user transfer.

2) Scheme pShare-: In this scheme, we choose $\alpha<1$ and without loss of generality we simulate $\alpha=0.5$.

3) Scheme pShare+: This scheme is a penalizing model where $\alpha>1$. H-op must pay to insure his client service. Without loss of generality, we choose $\alpha=1.25$ for the simulation.

\section{Simulation RESUlts}

\section{A. Simulation Setup}

We consider three cooperating operators, $O p_{1}, O p_{2}$ and $\mathrm{Op}_{3}$ each managing a single Radio Access Technology as in Fig.1. All managed RATs are supposed capable of delivering a constant value for the mean jitter $J_{M}$, mean delay $D_{M}$ and mean bit error rate $B E R_{M}$. The delivered parameters for each operator are depicted in Table I.

Users arrive in the system sequentially and we model the arrival and departure of users as a Poisson Process with mean arrival interval $1 / \lambda$ seconds. We perform simulation for different values taken from $1 / \lambda=\left[\begin{array}{llllll}6.67 & 5 & 4.44 & 4 & 3.63 & 3.33\end{array}\right.$ 3.076]. Once connected, the user will stay in the system for a certain service time, assumed to follow an exponential distribution of mean $1 / \mu=4 \mathrm{~min}$. During this service time, the user will consume a constant bit rate. Note that no scheduling is considered. And, at the end of the connection, the user will leave the system improving the available bandwidth of the serving operator. The simulations are done by Matlab for duration of 1200 seconds each and repeated for 70 experiments.

TABLE I. NETWORK PARAMETERS

\begin{tabular}{|l|l|c|c|c|}
\hline \multirow{2}{*}{$\begin{array}{c}\text { Network } \\
\text { operator }\end{array}$} & \multicolumn{4}{|c|}{ QoS Parameters } \\
\cline { 2 - 5 } Op1 & Bandwidth(Kb/s) & Jitter(ms) & Delay $(\boldsymbol{m s})$ & BER(dB) \\
\hline Op2 & 1700 & 6 & 19 & $10^{-3}$ \\
\hline Op3 & 11000 & 10 & 30 & $10^{-5}$ \\
\hline
\end{tabular}

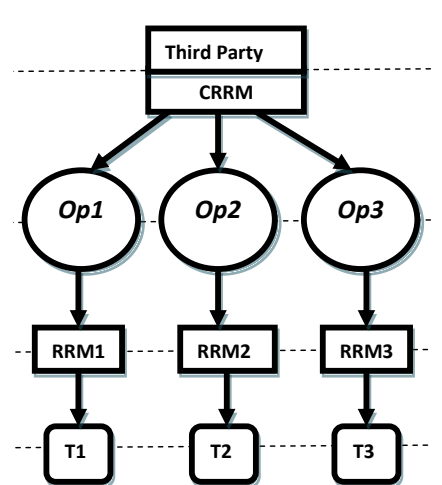

Fig. 1.Multi-operators mono-technology environment. 


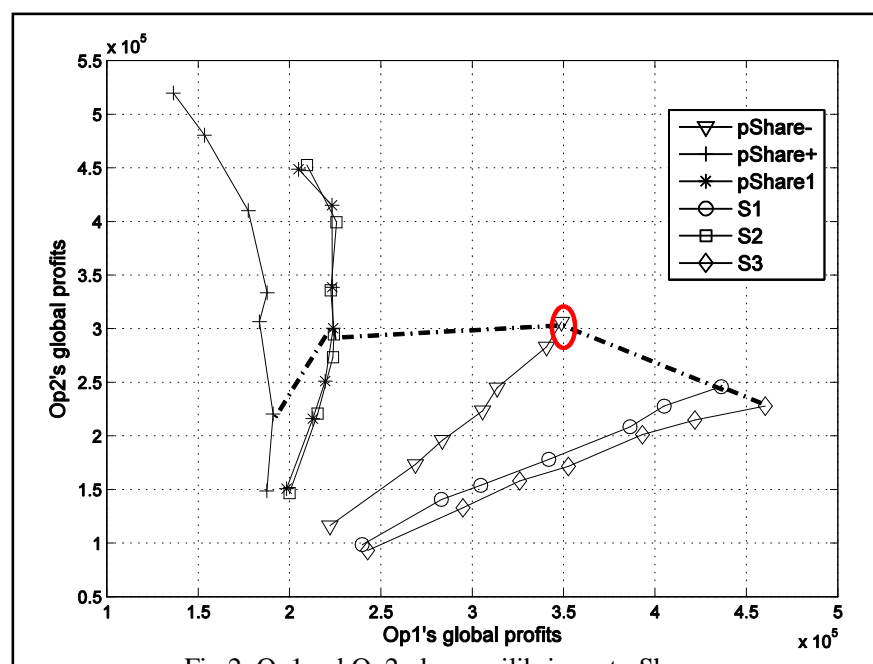

Fig 2. Op1and Op2 play equilibrium at pShare-.

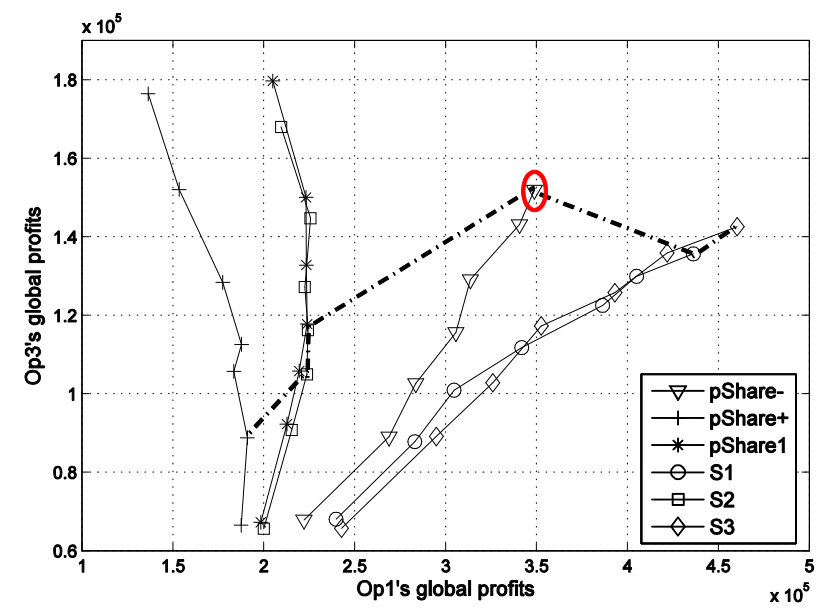

Fig 3. Op1 and Op3 play equilibrium at pShare-.

\section{B. Equilibrium pricing scheme}

The complexity of the Stackelberg game defined in section III arises from: the number of players (three operators) with two stages multi-leader follower play, the interchange of the leader follower roles (an operator can be a service operator in some states and a home operator transferring its user in another state) and the number of price strategies in stage I.

For simplification, we assume that all players in stage I adopt the same pricing scheme for the transaction cost settings. And the game solution is given as function of the adopted pricing scheme. The maximization problem in stage II is solved by simulation for each pricing strategy of stage II. Resulting payoffs (global achieved profits) for each players are taken from simulations output and plotted is the utility space $\left(\pi_{\mathrm{i}}, \pi_{\mathrm{j}}\right)$ to find the equilibrium strategy. Nash equilibrium solution is found for each pair of players in stage I. Nash equilibrium point gives the set of prices such that none of the operators can increase the profit by choosing a different price, given the price offered by the other service operator. The best response (equilibrium pricing scheme) is found for
Op1-Op2 play and Op1-Op3 play and Fig. 2 and 3 respectively. For every price strategy, we obtain a curve in the space $\left(\pi_{\mathrm{i}}, \pi_{\mathrm{j}}\right)$, we plot six curves corresponding to each pricing scheme. For each price $C s_{i}$ offered by the service operator $i$, the second player choose $C s_{j}$ in order to maximize its profit, such point corresponds to the tangent point of the vertical lines with the curve corresponding to $\mathrm{Cs}_{i}$. Connecting these points yields the dashed curve in Fig.2 and 3, representing the outcomes of the game for different prices. The best response of the game corresponds to the maximum point of the dashed curve.

Fig.2 represents the profit of $O p 2, \pi_{2}$, in function of the profit of $O p l, \pi_{1}$, for each strategy. For each strategy (pricing scheme) we find the points that maximize $O p 2$ payoff. Next, we connect these points and determine the maximum of the resulting curve. This maximum corresponds to the Nash equilibrium point where none of the operators can increase the profit by choosing a different price, without affecting the profit of the other player. The best response of $O p 1-O p 2$ game is reach with $p$ Share- scheme.

Fig.3 represents the profit of $O p 3, \pi_{3}$, in function of the profit of $O p 1, \pi_{1}$, for each strategy. Similarly, the best response of Op1-Op3 game is reach with $p$ Share- scheme.

Since both equilibriums are reached for the same pricing scheme, pShare- is adopted as the solution of $O p 1, O p 2$ and op3 multi-leader follower game.

\section{Selection game efficiency}

In the following, we study the system behavior with the best response pricing scheme pShare-. The efficiency of the selection game is shown through blocking rates improvement and the profits gains achieved via cooperation.

\section{1) Global blocking rates}

Figure 4 shows the improvement of the global blocking rates of the system formed by $O p 1, O p 2$ and $O p 3$. Results show that with cooperation, the access system is able to maintain a blocking rate below $2 \%$. Mobile users' acceptance is increase by $18 \%$ for high arrival rates.

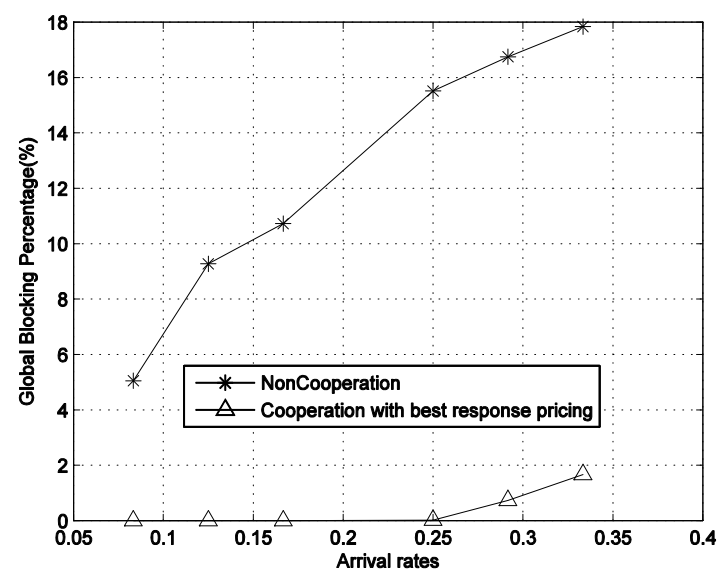

Fig 4. Global blocking rates reduction with cooperation. 


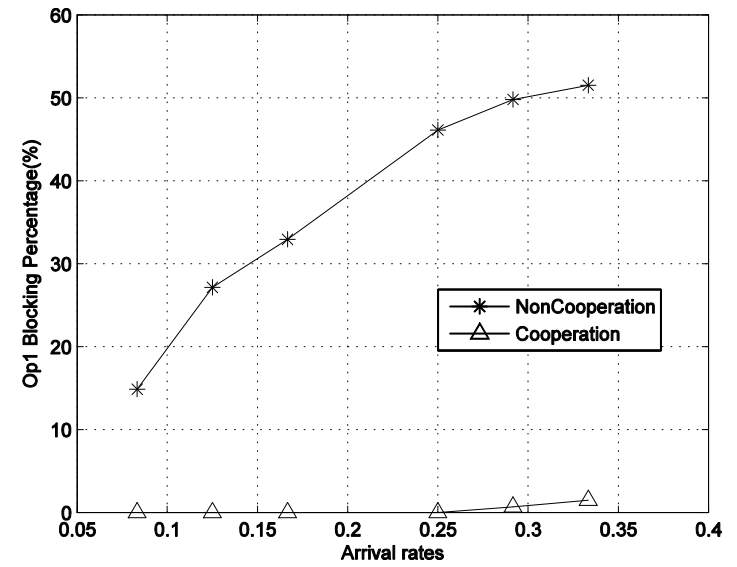

Fig 5a. Op1's network blocking rates improvement

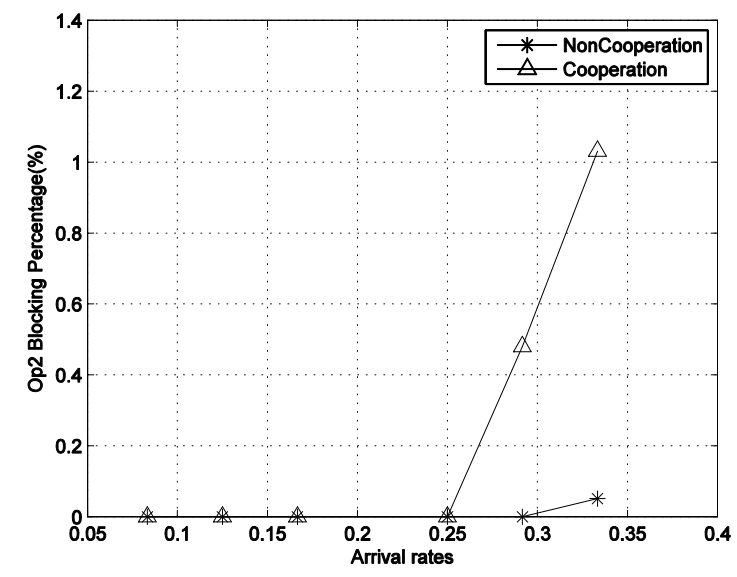

Fig 5b.Op2's network blocking rates conservation

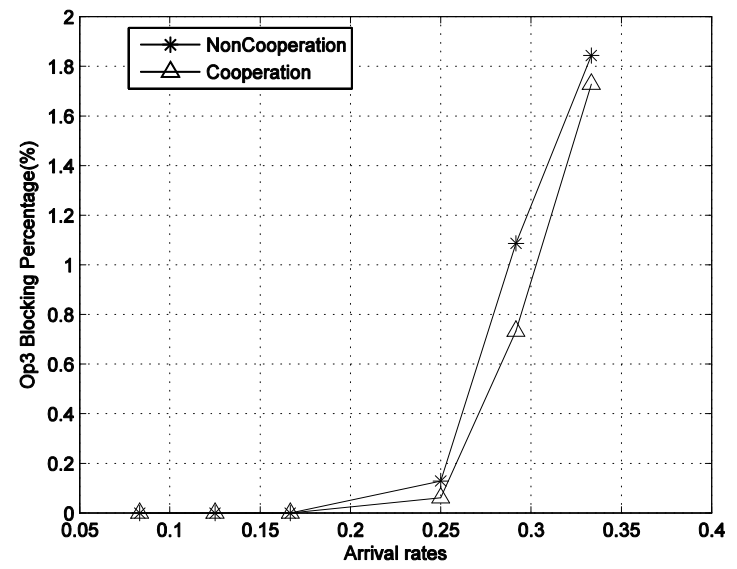

Fig 5c. Op3's network blocking rates improvement

2) Operators' blocking rates

Figure $5 \mathrm{a}, 5 \mathrm{~b}, 5 \mathrm{c}$ show the blocking rate variations after cooperation, for each operator of the system $O p 1, O p 2$ and $O p 3$ respectively. Operators could improve intensively their RAT blocking rates, especially the operators deploying a limited capacity as for Opl. The latter could improve the number of served users through cooperation by $50 \%$. Even

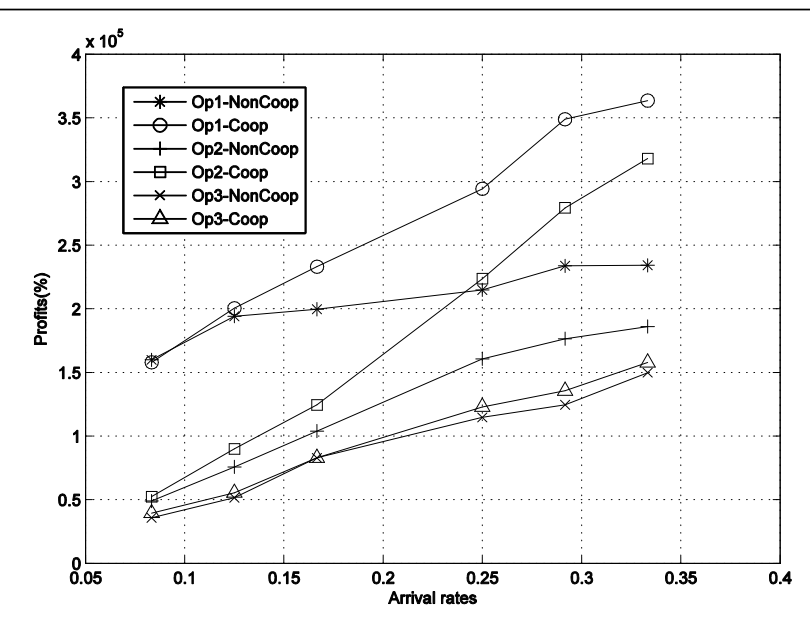

Fig 6.Achieved profits comparison

though, the operator deploys a high capacity, blocking rates are reduced and limited below $0.2 \%$, as for $O p 2$.

\section{3) Operators' profits}

Figure 6 shows profit variation for each operator before and after cooperation. In fact, the capacity gain achieved through operators' cooperation is translated into profit gain. New incomes are available from guests and transferred clients. The important increase of the user acceptance for $O p l$ brought more revenues. $O p 2$ also benefits from profit amelioration; additional incomes are achieved from serving guest users transferred from $O p 1$ and $O p 3$ networks. For $O p 3$ slight profit improvement is noticed because this operator exchanges users rarely. This operator benefits from cooperation explicitly, by avoiding resource underutilization.

\section{CONCLUSION}

Pricing in multi-operator wireless network is an important issue not only to maximize operators' revenue, but also to allocate resources efficiently. In this paper, we model the interaction between wireless operators as a multi-leaderfollower (Stackelberg) game. Cooperating service operators announce their transaction cost in the first stage and the home operator of the transferred user performs the selection decision in the second stage. The transaction cost price is set following six different predefined pricing schemes. The game solution is found using Nash equilibrium concept, and the best response is determine for every pairs of leaders. Results for three cooperating operators showed that sharing the half of the user payment solves the competition between the operators when one of the leaders is an operator with limited capacity and starves for cooperation to enhance its network performance.

Future works will upgrade this multi-leader-follower game with independent strategies. Dynamic pricing will be adopted where each player choses the service price independently of the other operator decision, in order to maximize their payoffs.

\section{REFERENCES}

[1] J. Markendahl, Mobile Network Operators and Cooperation: PhD thesis in telecommunication, Stockholm 2011,chap.3 pp.31-80. 
[2] K. Johansson, Cost Effective Deployment Strategies for Heterogenous Wireless Networks : Doctoral dissertation in telecommunication, Stockholm, November 2007.

[3] J. Markendahl, A. Ghanbari, "Shared Smallcell Networks, MultiOperator or Third Party- Or Both?", IWIOSC'13, Tsukuba Science City, Japan, May2013.

[4] J. Hultell, K. Johansson and J. Markendahl, "Business models and resource management for shared wireless networks", in Proc. IEEE VTC'04, Los Angeles, CA, USA, September 2004.

[5] A. Alsohaily and E.S. Sousa, "Performance gains of spectrum sharing in multi-operator LTE-advanced systems," in Proc. IEEE VTC'13 Fall, Las Vegas, USA, September 2013.

[6] A. Checko, H. L. Christiansen, Y. Yan, L. Scolaris, G. Kardaras, M. S. Berger and L. Dittman "Cloud RAN for mobile networks-a technology overview", IEEE Communications surveys \& tutorials, vol. PP, No. 99, September 2014.

[7] S. Farhat, A. Samhat and S. Lahoud, "Hybrid Decision Algorithm for Access Selection in Multi-operator Networks", in Proc. IEEE WCNC'14, Istanbul, Turkey, April 2014.

[8] S. Farhat, A. Samhat, S. Lahoud and B. Cousin,"Best operator policy in a heterogeneous wireless network", in SDIWC Proc. ICeND'14, Beirut, Lebanon, April-May 2014.

[9] L. Wang and G.S. Kuo, "Mathematical Modeling for Network Selection in Heterogenuous Wireless Networks-A Tutorial", IEEE Communications serveys\& tutorials 2011.

[10] Q.S. Nguyen-Vuong, Y. Ghamri-Doudane and N. Agoulmine, "On utility models for access network selection in wireless heterogeneous networks", in Proc. Network Operations and Manage. Symp. (NOMS), 2008, pp. 144-151.

[11] O. Ormond, J. Murphy and G.M. Muntean, "Utility-based intelligent network selection in beyong 3G systems", in Proc. IEEE ICC, 2006, pp. 1831-1836.

[12] F.W. Karam, and T. Jensen, "A QoS based Decision Algorithm in next generation networks," in Proc. IEEE ICCM'12, Seoul, Korea, April 2012.

[13] G. Fortetsanakis, M. Papadopouli, G. Karlsson, M.Dramitinos, E.A. Yavuz, "To subscribe, or not to subscribe: Modeling and analysis of service paradigms in cellular markets", in Proc. IEEE DYSPAN'12, Bellvue, USA, October 2012.

[14] L.Giupponi, R. Agusti, J. Pérez-Romeo and O. Sallent, "Improved revenue and radio resource usage through inter-operator joint radio resource management," in Proc. IEEE ICC'07, Glasgow, Scotland June 2007.

[15] L.Giupponi, R. Agusti, J. Pérez-Romeo and O. Sallent, "Inter-operator agreements based on QoS metrics for improved revenue and spectrum efficinecy", Electronics Letters Vol.44 No.4, February 2008.

[16] E. Stevens-Navarro and V. Wong, "Comparison between vertical handoff decision algorithms for heterogenous wireless networks," in Proc. IEEE VTC, May 2006.

[17] X.Cao, H. Shen, R. Miloto and P. Wirth, "Internet Pricing with Game Theoretical Approach: Concepts and Examples", IEEE/ ACM Transactions on Networking, Vol. 10, April 2002.

[18] D. Niyato, E. Hossain,"Competitive pricing in heterogeneous wireless access networks: issues and approaches," IEEE Network, November/December 2008.

[19] J. Elias, F. Martignon, L. Chen and E. Altman, "Joint Operator Pricing and Network Selection Game in Cognitive Radio Networks: Equilibrium, System Dynamics and Price of Anarchy", IEEE Transaction on Vehicular Technology, vol. 62, No. 9, November 2013.

[20] V. Gajié, J.Huang and B. Rimoldi, "Competition of Wireless Providers for Atomic Users", IEEE/ACM Transactions on Networking ,2013. 